\title{
Vacuum bell therapy
}

\section{Frank-Martin Haecker, Sergio Sesia}

Department of Pediatric Surgery, University Children's Hospital, Basel, Switzerland

Correspondence to: Frank-Martin Haecker, MD, Professor of Pediatric Surgery, FEAPU. Department of Pediatric Surgery, University Children's Hospital, Spitalstrasse 33, 4031 Basel, Switzerland. Email: frankmartin.haecker@ukbb.ch.

Background: For specific therapy to correct pectus excavatum (PE), conservative treatment with the vacuum bell $(\mathrm{VB})$ was introduced more than 10 years ago in addition to surgical repair. Preliminary results using the VB were encouraging. We report on our 13-year experience with the VB treatment including the intraoperative use during the Nuss procedure and present some technical innovations.

Methods: A VB with a patient-activated hand pump is used to create a vacuum at the anterior chest wall. Three different sizes of vacuum bells, as well as a model fitted for young women, exist. The appropriate size is selected according to the individual patient's age and ventral surface. The device should be used at home for a minimum of 30 minutes (twice a day), and may be used up to a maximum of several hours daily. The intensity of the applied negative pressure can be evaluated with an integrated pressure gauge during followup visits. A prototype of an electronic model enables us to measure the correlation between the applied negative pressure and the elevation of the anterior chest wall.

Results: Since 2003, approx. 450 patients between 2 to 61 years of age started the VB therapy. Age and gender specific differences, depth of PE, symmetry or asymmetry, and concomitant malformations such as scoliosis and/or kyphosis influence the clinical course and success of VB therapy. According to our experience, we see three different groups of patients. Immediate elevation of the sternum was confirmed thoracoscopically during the Nuss procedure in every patient.

Conclusions: The VB therapy has been established as an alternative therapeutic option in selected patients suffering from PE. The initial results up to now are encouraging, but long-term results comprising more than 15 years are so far lacking, and further evaluation and follow-up studies are necessary.

Keywords: Pectus excavatum (PE); conservative treatment; vacuum bell (VB)

Submitted Mar 10, 2016. Accepted for publication Jun 06, 2016.

doi: 10.21037/acs.2016.06.06

View this article at: http://dx.doi.org/10.21037/acs.2016.06.06

\section{Introduction}

Before the turn of the millennium, childhood and adolescent pectus excavatum (PE) was mainly treated surgically through the open Ravitch technique (1). In 1998, Nuss et al. reported their 10-year experience in using a minimally-invasive technique for pectus excavatum repair (2). Today, the minimally-invasive repair of pectus excavatum (MIRPE) is well established and is the 'goldstandard' for surgical repair of PE in adolescents (3-8). However, MIRPE is operator-dependent and has a number of potential complications, which have been widely reported in the literature (3-14).
Conservative treatment of $\mathrm{PE}$ through the application of a glass bell to elevate the sternum to correct was first described over 100 years ago, which is called the vacuum bell (VB) (15). In addition, patients were recommended to use intensive inspiration and expiration, as well as the application of special dressings. Despite the ongoing risks and/or unsatisfactory results after operative therapy for some patients, the routine use of the VB technique could not be established. This was because of the inadequacy of the glass bell material and its difficult application to the ventral chest wall. In 1992, the engineer E. Klobe, who suffered from PE, developed a new device to conservatively treat PE (16). Using this VB through a period of 2.5 years, 


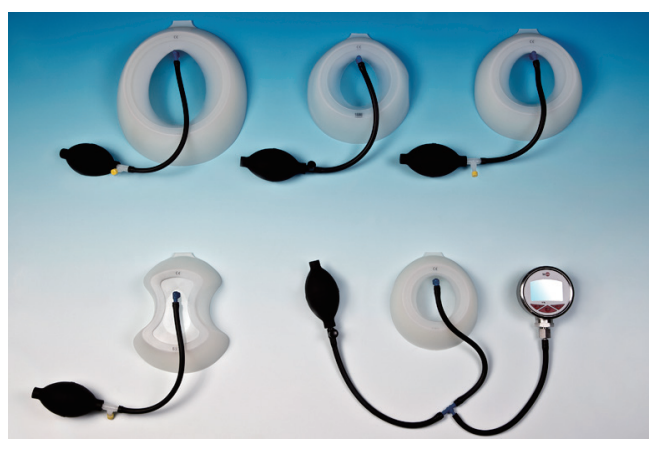

Figure 1 Different models of the vacuum bell (medium size model with integrated measurement device).

he was able to elevate the sternum and to correct his PE without any visible deformities (16).

Initial results from pilot studies describing the use of VB therapy in PE patients were published 10 years ago and proved to be promising $(17,18)$. CT-scans showed that the device was able to lift the sternum and ribs immediately (17). Afterwards, several studies involving a larger pool of patients were done $(19,20)$, and the first systematic review summarizing the results of 133 patients was published in 2011 (21). Furthermore, recent studies confirmed the efficacy of VB therapy in carefully selected patients (22-24).

In this paper, we report on our 13-year experience in using the VB, which includes the results of a pilot study evaluating its intraoperative use during MIRPE. Additionally, we will present some technical innovations that have enabled us to record the effect of VB therapy quantitatively and qualitatively.

\section{The vacuum bell}

Technical details of the VB as well as instructions concerning the application of the VB have been described in the literature $(16,21,22,24)$. There are three different sizes of the VB (16, 19 and $26 \mathrm{~cm}$ in diameter), which are chosen according to the individual patients age and ventral surface. The medium-sized model is available in a supplemental version with a reinforced silicon wall (type "bodybuilder"), which is suited for adult patients with a small, deep PE. For adolescents and adult female patients, a special fitted model is available (Figure 1) (16). The VBs by E. Klobe are CE certified and patent registered. In the United States, the device was approved by the food and drug administration (FDA) in May 2012.

According to the instructions and our experience, the VB should be used at home for a minimum of 30 minutes, twice a day during 4-6 weeks. Afterwards, the application may be used up to a maximum of several hours daily $(16,18-22)$. The immediate elevation of sternum and ribs during application of the VB was demonstrated through a study by Schier and Bahr (17). We confirmed this effect by thoracoscopy during the MIRPE procedure (25). We use the $\mathrm{VB}$ routinely in every patient during MIRPE.

\section{Indications, contraindications and side effects}

In general, VB therapy is feasible at nearly any age. Patients age and patients compliance as well as the ventral surface for local application are important factors. We believe that VB therapy is indicated in patients who present with mild $\mathrm{PE}$ and/or wish to avoid surgical procedure. Contraindications to VB include musculoskeletal disorders, vasculopathies, coagulopathies and cardiac disorders (16, 18-22,24). To exclude these disorders, a standardized evaluation protocol is routinely performed before beginning the therapy.

Complications include subcutaneous hematoma, petechial bleeding, dorsalgia and transient parasthesia of the upper extremities during application of VB $(16,21,22)$.

\section{Methods}

Patients are referred by pediatricians, general practitioners or orthopaedic surgeons to our specialized outpatient clinic for chest wall deformities. Furthermore, we are seeing an increasing number of patients who have referred themselves. All PE patients are informed about the option of conservative versus surgical therapy to correct PE. Standardized evaluation includes patient and family history, clinical examination, measurement of depth of PE [in a standardized supine position using a designed scaled rod, which is adapted to the different available models of the $\mathrm{VB}$ (Figure 2)], and photo documentation. Contraindications must be excluded. To exclude cardiac anomalies, cardiac evaluation is routinely performed using electrocardiogram and echocardiography prior to daily VB application.

The first application of the VB occurs during the outpatient clinic visit under supervision of the attending physician. The appropriate size and model of the different type is selected according to patient age, ventral surface of the chest wall, and patient self-perception of the deformity. Patients learn the proper application of $\mathrm{VB}$, having the middle of the window positioned above the deepest point of 


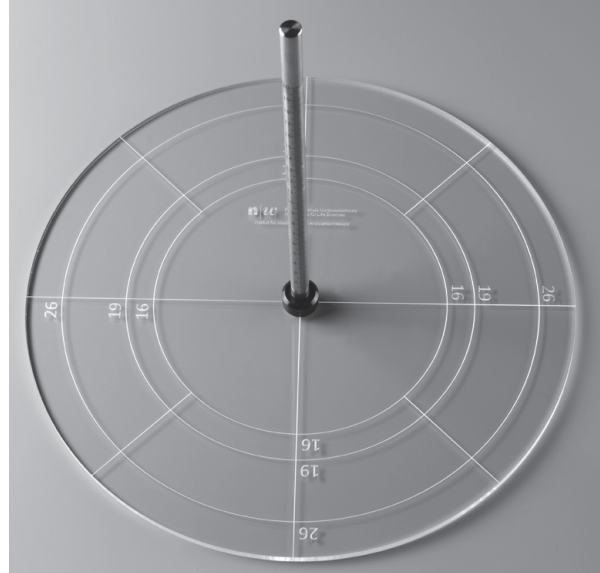

Figure 2 Scaled rod adapted to the available vacuum bell models for standardized measurement of pectus excavatum.

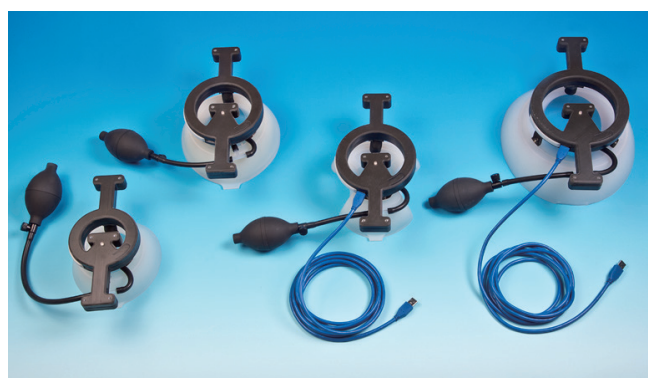

Figure 3 Different models of the vacuum bell with integrated electronic measurement device.

the PE. Parents or caregivers should supervise application of $\mathrm{VB}$ in children under 10 years $(16,19-22,24)$. In patients with an asymmetric $\mathrm{PE}$ or a grand canyon type $\mathrm{PE}$, it could be useful to apply the device in different positions. In patients with a localized deformity, it could be helpful to use a small sized VB.

After exclusion of cardiac anomalies and other contraindications, patients may start with the daily application. All users are recommended to use the device twice daily for 30 minutes each for the first 4-6 weeks. Later on, the length of time of daily application of the VB will vary widely between patients. Some of the adult patients use the VB up to 8 hours daily during office hours, whereas adolescent boys may apply the device every night for 7-8 hours. In our experience, the duration and frequency of daily application depends on each patient's individual choice and motivation.

Patients undergo follow-up visits at 3 to 6 monthly

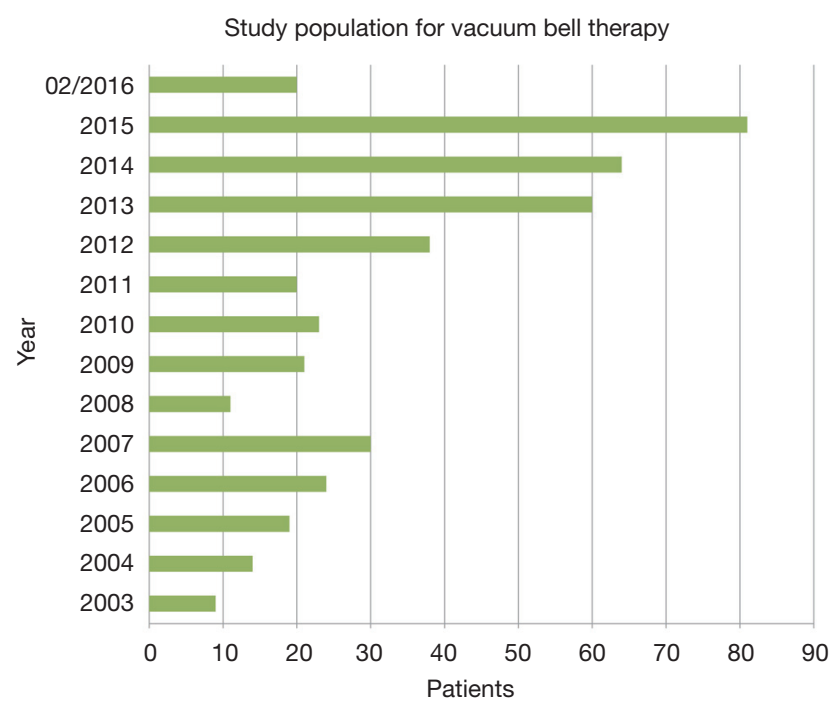

Figure 4 Number of new vacuum bell patients per year.

intervals, including clinical examination, measurement of depth of PE and photo documentation. In our outpatient clinic, we may evaluate the intensity of the applied negative pressure with an integrated pressure gauge. Furthermore, the correlation between the applied negative pressure and the elevation of the anterior chest wall can be assessed with our electronic VB model (Figure 3). Tips and tricks to optimize the application will be discussed. Clinical examination focuses on the improvement of depth of $\mathrm{PE}$ as well as side effects such as persistent hematoma and/or skin irritation. The endpoint of therapy is defined by each patient's individual decision, which is confirmed by clinical examination during outpatient clinic visit.

\section{Results}

Within the last 13 years, 434 patients ( 82 female, 352 male) started with VB treatment at our institution (Figure 4). The median age was 16.2 years (2-61 years). Upon commencing VB therapy, 113 patients were over the age of 18; however, the majority of our patients were children and adolescents (Figure 5). The first retrospective study not only described the method, but also assessed its efficacy by systematically including a subset of our patients group that was published in 2011 (21). Updated and more detailed results, which include another subset of patients, have been summarized in a previously published study (22).

Patient perceptions to therapy are different, and it is highly dependent on the applicant's age. As mentioned 


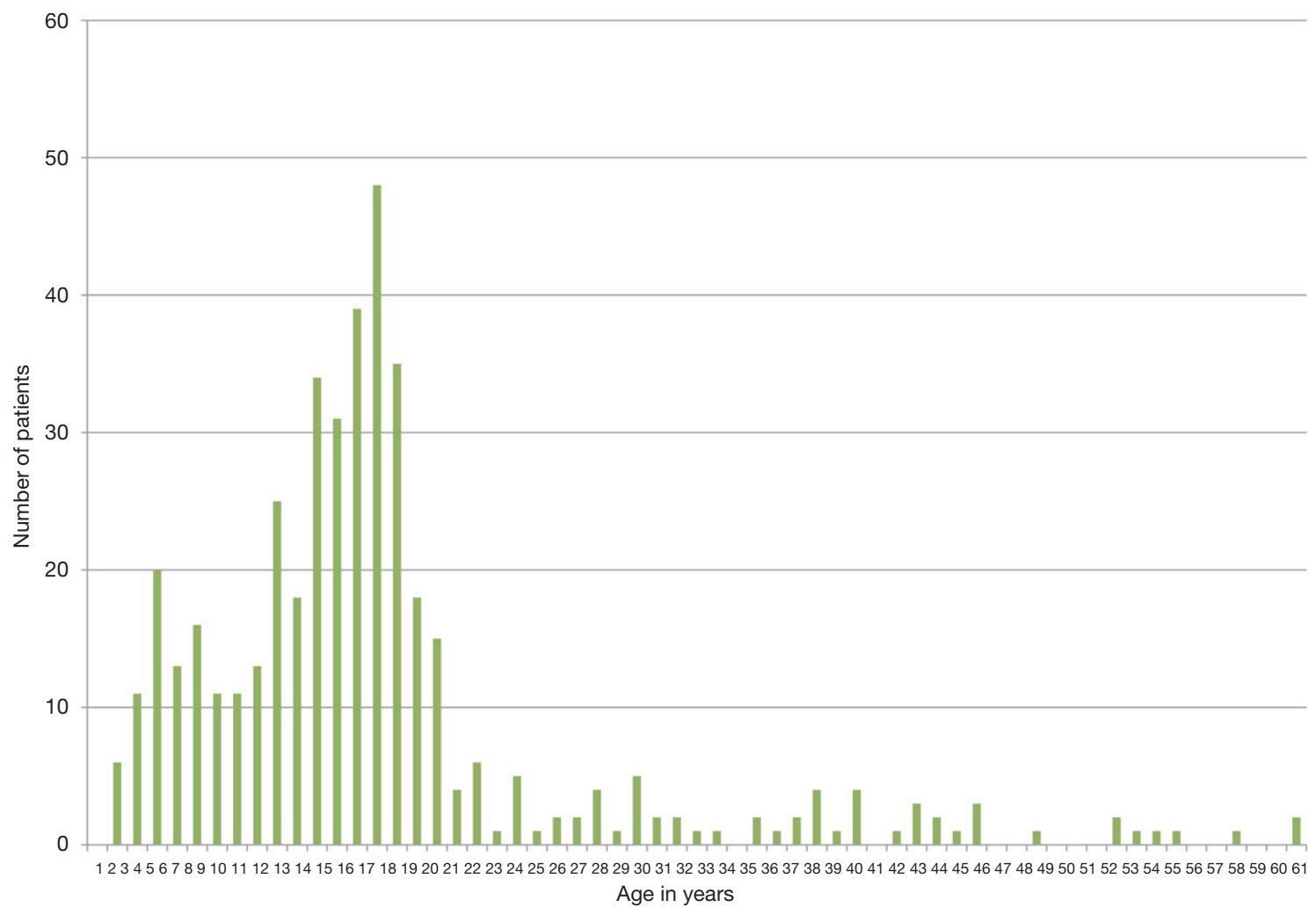

Figure 5 Age distribution of vacuum bell patients.

previously, we observed age-specific differences in success $(16,21,22)$. During the first five applications, most of the patients experienced moderate pain in the sternum and report a feeling of uncomfortable pressure within the chest. Adolescents and older patients can develop moderate subcutaneous hematomas, which disappear within a few hours. Some patients report temporary side effects, such as transient paresthesia of the upper extremities during the application and/or mild dorsalgia. These symptoms disappear when lower atmospheric pressure is used during application. Analgesic medication is usually unnecessary, and its usage was not reported by any patients or parents. As mentioned above, parents or caregivers should supervise application of VB in children under 10 years. In this age group, no relevant side effects were reported.

Our latest published study (22) included a subset of 140 patients (112 males, 28 females), aged 3 to 61 years (median 16.05 years). This is a retrospective study of a prospectivelykept database with consecutive patient recruitment. VB was used for 6 to maximum 69 months (average 20.5 months). Before starting with VB therapy, patients presented with a $\mathrm{PE}$ with depth ranging from 1-6.3 $\mathrm{cm}$ (average $2.7 \mathrm{~cm}$ ). Daily application of $\mathrm{VB}$ in the whole group was 107.9 minutes/day (range, 10-480 minutes). VB therapy was terminated after 20.5 months. In 61 patients, the sternum was lifted to a normal level after 21.8 months (range, 6-69 months) (Figures 6,7). The follow-up period was 27.6 months after discontinuation of VB therapy (range, 1-73 months), without any regression to $\mathrm{PE}$ until today (Figures 6,7). Fifty-four patients were still under going treatment. Every patient was satisfied and expressed interest in continuing the therapy. They were motivated and compliant, which is paramount to achieving a successful therapy. However, 25/140 patients stopped the application after 15.7 months (range, 1-42 months), due to an unsatisfactory result and/or decreasing motivation. A total of $15 / 25$ patients underwent MIRPE. Notable side effects within the study group were local skin irritation (13.6\%), localized pain during application (12.1\%) and hematoma at the deepest point of $\mathrm{PE}(7.1 \%)$.

The importance of patient motivation is illustrated by the fact that 15 patients who underwent MIRPE used the VB for 160.6 minutes/day, whereas the remaining 10 patients who stopped the therapy have only used the VB for 36.3 minutes/day. In three patients with asymmetric 

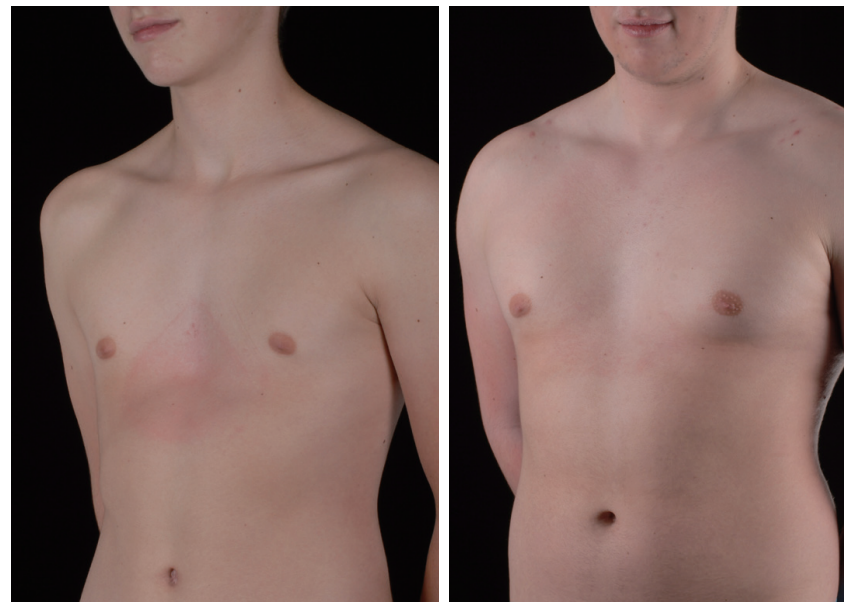

Figure 6 Sixteen-year-old patient, before (left: depth of PE = $2.3 \mathrm{~cm}$ ) VB therapy and after 24 months (right: depth of $\mathrm{PE}=$ $1.3 \mathrm{~cm}, 6$ months after cessation of VB therapy)
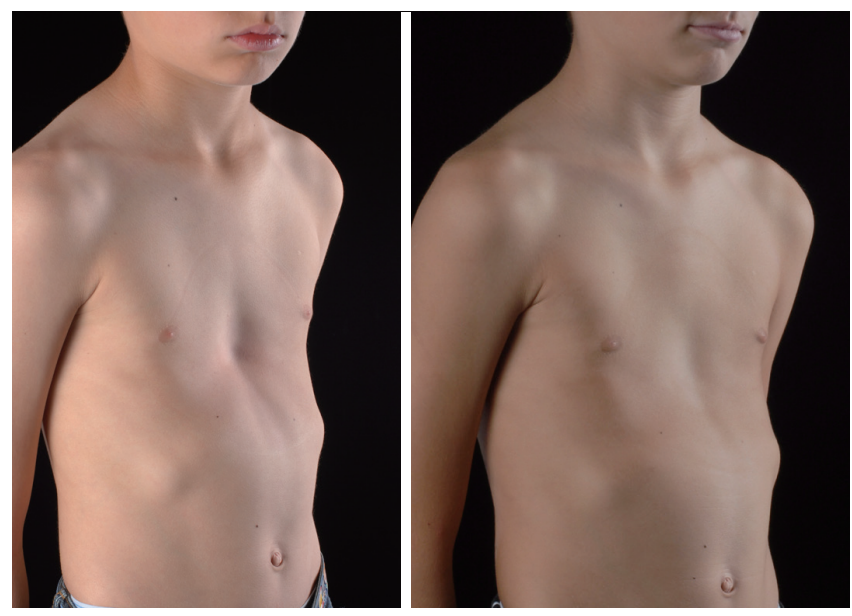

Figure 7 Ten-year-old boy, before (left: depth of $\mathrm{PE}=2.2 \mathrm{~cm}$ ) VB therapy, and 12 months after cessation of VB therapy (right: depth of $\mathrm{PE}=0.5 \mathrm{~cm}$ ); duration of therapy: 36 months.

$\mathrm{PE}$, the depth of PE has decreased after 9 months, but the asymmetry is still visible.

All our patients were recommended to continue sporting activities and physiotherapy in order to improve body control, which is an important factor in patient outcomes.

We know from our retrospective studies that factors such as age and gender specific differences, depth of PE, symmetry or asymmetry, and concomitant malformations like scoliosis and/or kyphosis, may influence the clinical course and the success of this therapy. However, we could not determine which factor is the most influential. Therefore, we are not able to predict when VB therapy will be successful or contraindicated in correcting PE. It would be useful to have reliable parameters to include or to exclude patients from VB therapy for patients, their parents and physicians.

However, we can draw a number of conclusions. Firstly and most importantly, the optimal age for this treatment has yet to be defined. In our experience, the growth spurt during puberty is the most important period to influence degree and depth of PE. It is important for future studies to evaluate whether commencing VB therapy before puberty will be more useful than starting during puberty or later. Our preliminary results recommend starting VB therapy before the age of 10 years. Furthermore, long-term results for patients that are 15 years and more are still unavailable. Further follow-up studies are necessary to elucidate these facts. Second, patients or their parents will have to pay directly to obtain the VB in most European countries. In some countries in South America, the purchasing of VB is covered by the individual national health care system or the local insurance.

Physicians and patients will have to discuss the benefits and risks of using the VB prior to MIRPE. As the majority of patients will have to pay for the device in our country, most of our patients are not interested in this "pretreatment". Additionally, we observed no significant difference in outcomes between patients who had VB therapy prior to surgery and those who did not (25).

According to our experience based on our study group, we see three different groups of patients:

(I) PE-patients (children to pre-adolescents) with a mild, symmetric PE (depth $<3 \mathrm{~cm}$ ) with a flexible chest wall; the duration of treatment is expected to be 12 to 15 months.

(II) PE-patients (adolescents to adults) with a moderate $\mathrm{PE}$ (depth of $\mathrm{PE}>3 \mathrm{~cm}$ ) with a less flexible chest wall; these patients need close and careful monitoring every three months; the duration of treatment is expected to be 24 to 36 months.

(III) PE-patients with a severe (asymmetric) PE with a stiff chest wall, who initially presented during their pubertal growth spurt; these patients represent the "risk group" for failure of the conservative treatment.

We initiated a number of pilot studies to corroborate our hypothesis and evaluate different aspects of the treatment (see below). 


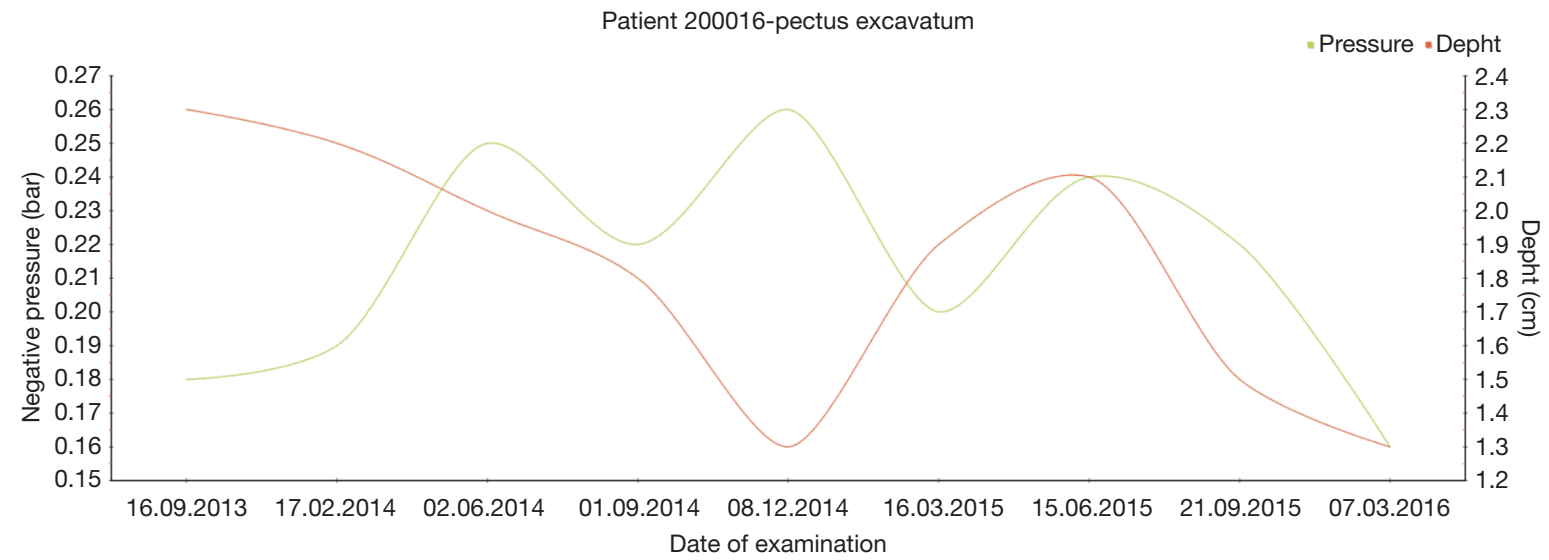

Figure 8 Assessment of applied pressure in correlation to the depth of PE (16-year-old boy).

\section{Pilot studies evaluating the effectiveness of vacuum bell therapy Quantitative measurement of applied negative pressure}

The success of a therapeutic procedure not only requires a good technique, but also its use for an appropriate indication. In addition to clinical examination and measurement of depth of PE, we considered making use of a quantitative assessment of the negative pressure necessary to lift the sternum during the first application. This measurement would enable us to stratify patients into different groups, identify suitable patients, and allow us to accurately predict those who will benefit from this method and those who will not. Since 24 months, we have been using an integrated device to measure the applied negative pressures (Figure 1). In a group of 148 patients [median age 13.17 years, 2 to 61 years, standard deviation $(\mathrm{SD}) \pm 9.0$ ears], the negative pressure during the first application was 0.122 bar (0.01 to 0.2 bar; $S D \pm 0.061$ bar). During followup visits, we first observed an increase in negative pressure, then a decrease in negative pressure following cessation of VB use. Monitoring the applied negative pressure in correlation to the depth of PE shows successful treatment or failure (Figures 8,9). Furthermore, we can confirm the correlation between patient age and the negative pressure required to elevate the sternum. The ongoing clinical trial at our institution will enable us to provide a more detailed analysis, including follow-up data from our database.

\section{Objective assessment of sternal elevation in correlation to the applicated pressure}

Two years ago, we developed a prototype of an electronic device in collaboration with engineers at our technical university to assess the elevation of the chest wall in correlation to the applied negative pressure. Initial results proved to be promising (26). After IRB approval, we started a pilot study evaluating appropriate patients in our specialized outpatient clinic. This new device enables us to measure the sternal elevation in correlation to the applied negative pressure in every patient. We may assess agespecific differences and monitor these assessments during follow-up visits (Figures 9-11). In an example of a 3-year-old patient, postoperatively we can see an immediate elevation of the sternum as well as increased breathing-dependent movement of the ventral chest wall as an indirect sign of high flexibility (Figure 9). In contrast, we see a slower increase of the negative pressure and less elevation of the sternum in a 19-year-old patient (Figure 10). Figure 11 shows the results in a 61-year-old patient.

\section{Pulmonary function (PF)}

The influence of $\mathrm{PE}$ on $\mathrm{PF}$ is highly controversial within the literature. However, there are numerous studies that report a significant improvement of $\mathrm{PF}$ after surgical repair of PE. To the best of our knowledge, there is currently no data available concerning $\mathrm{PF}$ tests in conservative treatment of patients with PE. In collaboration with the department of paediatric pulmonology at the UKBB, we started a prospective study titled "Lung function in chest wall and spine deformities-value of oscillometry techniques and longitudinal development with growth". We will follow-up PE-patients who use the VB for at least 12 to 15 months.

\section{Intraoperative use}

With widespread use of MIRPE today, the frequency of 


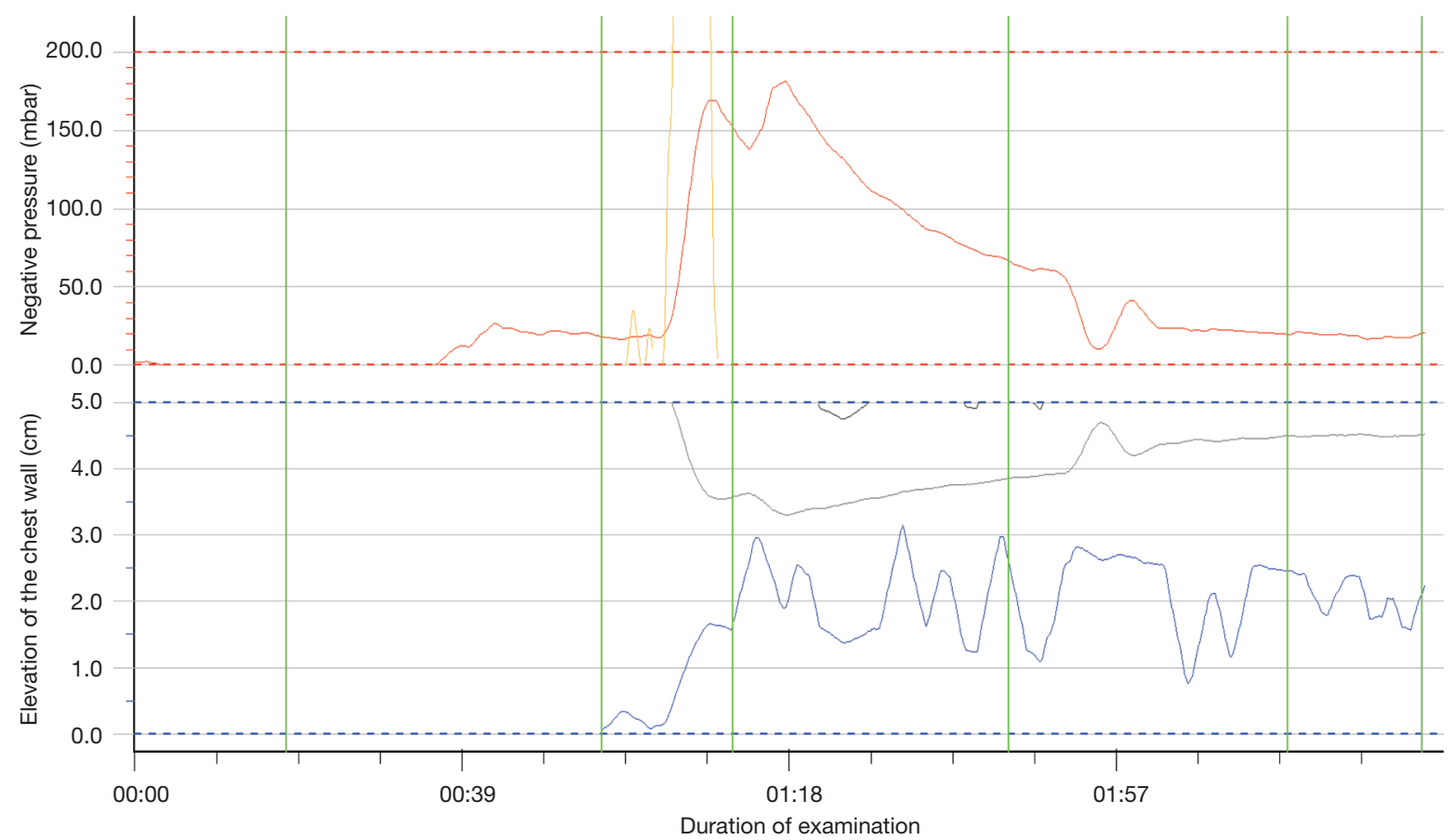

Figure 9 Electronic assessment of VB application: examination of a 3-year-old patient. [red: negative pressure (in mbar); blue: elevation of the chest wall (in cm); yellow: activation of the hand pump].

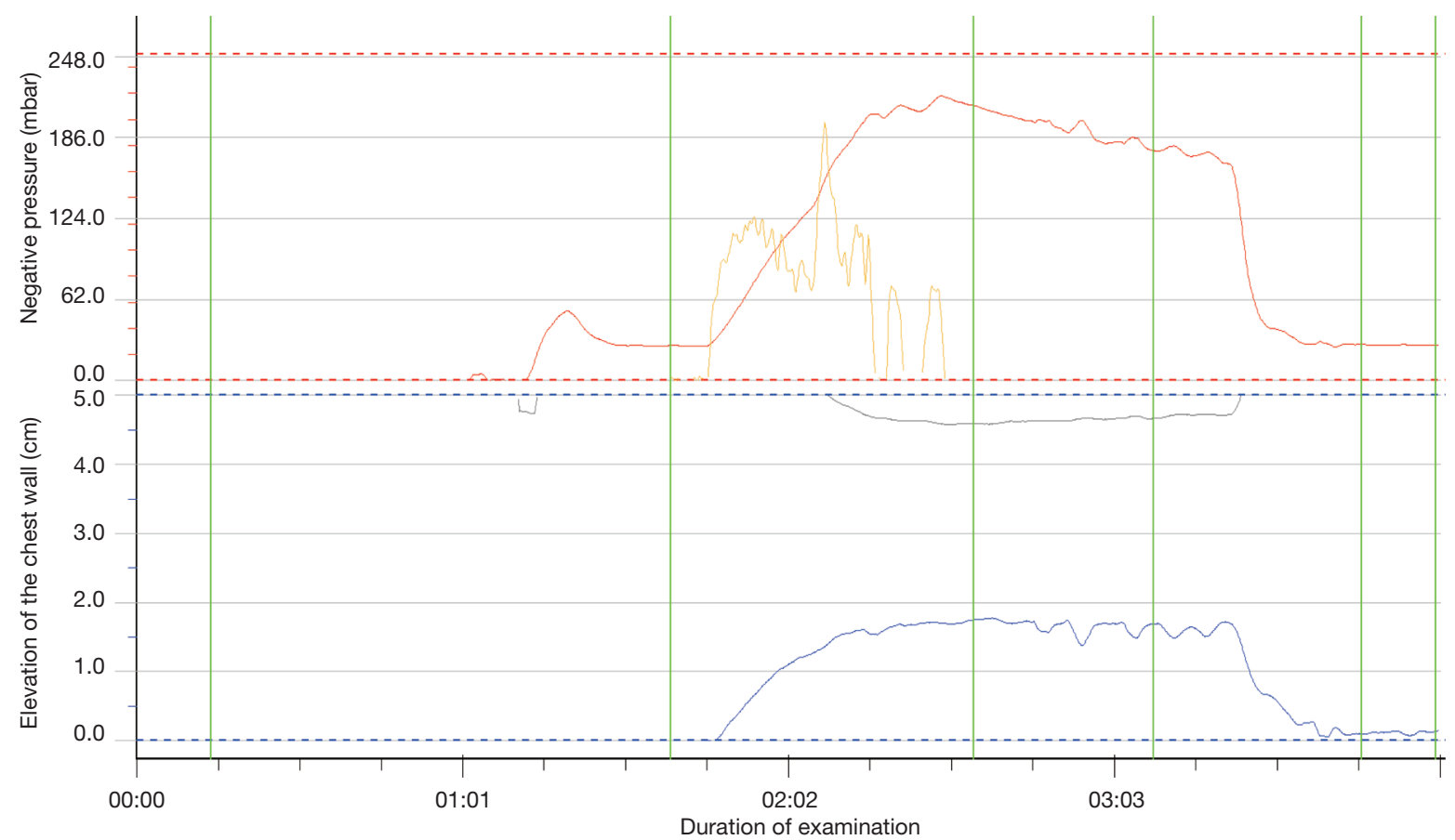

Figure 10 Electronic assessment of VB application: examination of a 19-year-old patient. [red: negative pressure (in mbar); blue: elevation of the chest wall (in cm); yellow: activation of the hand pump]. 


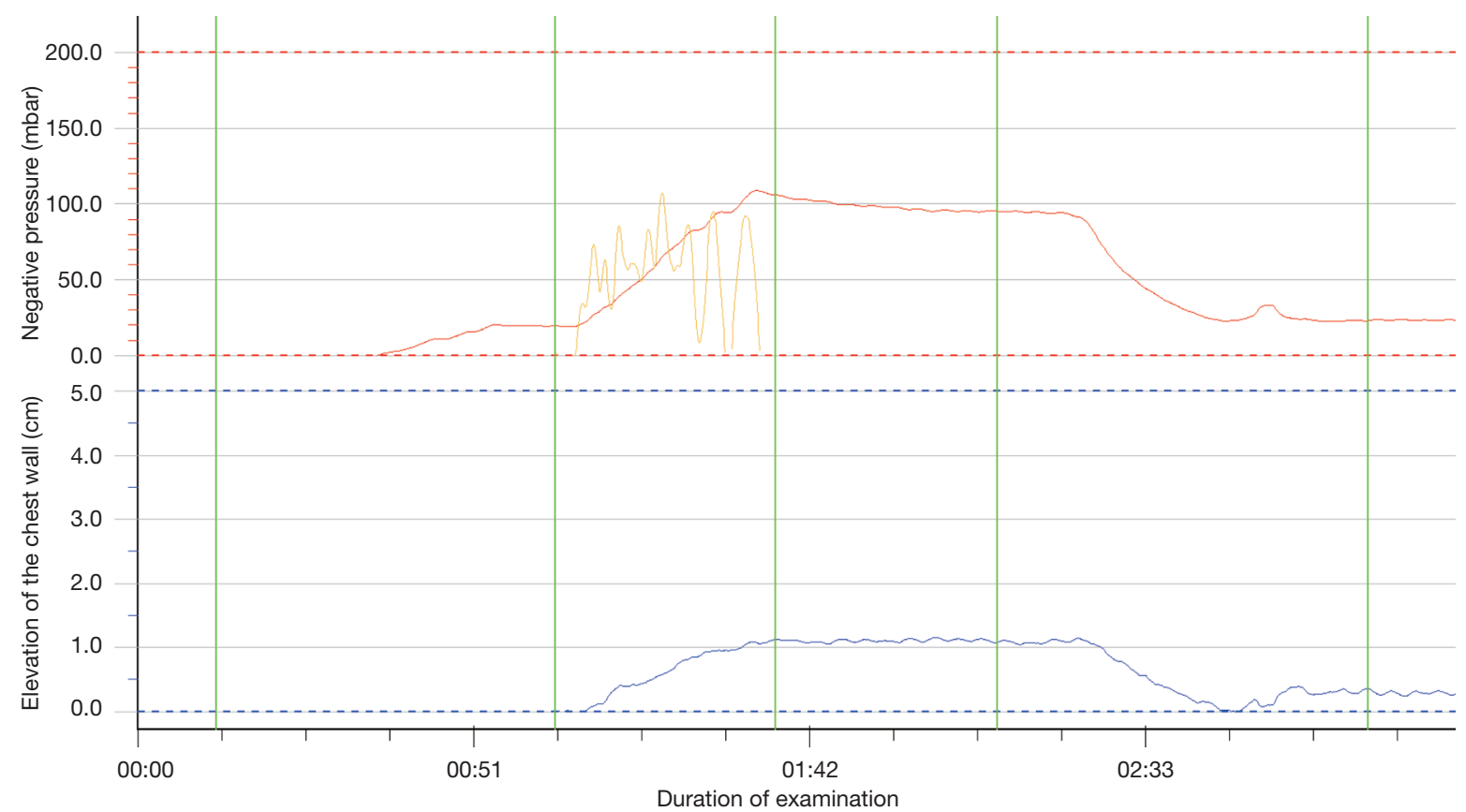

Figure 11 Electronic assessment of VB application: examination of a 61-year-old patient (red: negative pressure (in mbar); blue: elevation of the chest wall (in cm); yellow: activation of the hand pump).

complications has increased. Numerous studies reported an increasing number of near-fatal complications, such as injury to the heart and the mammary vessels. To avoid these complications, the routine use of intraoperative thoracoscopy was established years ago. Furthermore, several techniques to elevate the sternum during retrosternal dissection have gained wide acceptance. Above all, surgeons who operate on adult patients presenting with a deep PE and a stiff chest wall prefer to use a crane or a special clamp to elevate the sternum intraoperatively $(5,6,10)$. Schier and Bahr demonstrated in their pilot study that, when creating the vacuum, the elevation of the sternum is obvious (17). Based on this experience and the preliminary results from our first study (18), we speculate that VB may also be useful in reducing the aforementioned risks of injury during the MIRPE procedure.

Since 2005, we have been using the device intraoperatively during MIRPE to facilitate the dissection of the transmediastinal tunnel and the advancement of the pectus introducer, the riskiest step of the procedure. The device may also be applied for placement of the pectus bar. However, the manufacturer of the device does not have a license for sterilisation of the VB; therefore, the use of the device for placement of the pectus bar is considered "offlabel". In agreement with our hospital hygienist and bearing in mind the nature of the material, we used gas sterilization for preparation of the device intraoperatively.

In our pilot study, 50 patients aged from 9 to 28 years (average 14.95 years) were included. They were operated on for PE using the MIRPE procedure. Thirty-eight patients underwent primary surgery. Twelve patients used the vacuum bell for a period of 4-36 months (average 19.9 months) before surgery, and discontinued the application due to decreased motivation and/or insufficient success. The VB was applied for retrosternal dissection and advancement of the pectus introducer, as well as the placement and flipping of the pectus bar. The use of the VB led to a clear elevation of the sternum, which was confirmed by thoracoscopy (Figure 12). Advancement of the pectus introducer and placement of the pectus bar is deemed to be safe. All patients develop a mild postoperative hematoma, which resolves itself during the hospital stay. No evidence of cardiac and/or pericardial lesions or lesions of the mammary vessels were noted intraoperatively through right-sided thoracoscopy. Additionally, no midline incision to elevate the sternum with a hook was necessary (25).

\section{Limitations}

Since age-specific differences were significant contributors 

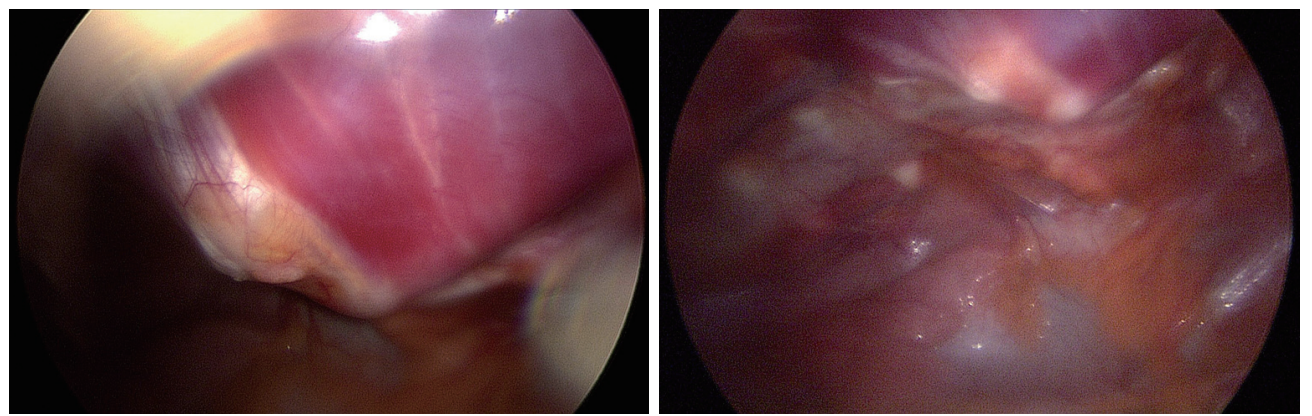

Figure 12 Intraoperative use of the VB (left: before application, right: during application).

of success in our large study population, the optimal age for this treatment has still to be defined. Although we developed a thorough protocol for follow-up visits every 3 months, home surveillance of VB therapy must be optimized. Additional electronic devices may enable us to supervise the duration and intensity of daily application at home. Furthermore, the time of follow-up with a maximum of 10 years is found to be insufficient in confirming the long-term effect of $\mathrm{VB}$ therapy.

\section{Conclusions}

Within the last 10 years, VB therapy has been established as a beneficial tool in the treatment of patients suffering from PE. VB therapy may allow some patients with PE to avoid surgery. In our experience, patients with symmetric and mild PE are likely to benefit from this procedure. The application is easy, and is accepted well by both pediatric and adult patients. However, the optimal age for this treatment has yet to be defined. In our experience, the growth spurt during puberty is the most important period to influence degree and depth of PE. It is important for future studies to evaluate whether initiating VB therapy before puberty will be more useful than starting during or after puberty, as well as the long-term effects of this therapeutic tool. The results of ongoing studies will allow us to answer at least some of these questions.

The intraoperative use of VB during the MIRPE facilitates the introduction of the pectus bar. In any case, the method seems to be a valuable adjunct therapy in the treatment of PE.

\section{Acknowledgements}

None.

\section{Footnote}

Conflicts of Interest: The authors have no conflicts of interest to declare.

\section{References}

1. Ravitch MM. The Operative Treatment of Pectus Excavatum. Ann Surg 1949;129:429-44.

2. Nuss D, Kelly RE Jr, Croitoru DP, et al. A 10-year review of a minimally invasive technique for the correction of pectus excavatum. J Pediatr Surg 1998;33:545-52.

3. Haecker FM, Bielek J, von Schweinitz D. Minimally invasive repair of pectus excavatum (MIRPE)--the Basel experience. Swiss Surg 2003;9:289-95.

4. Hosie S, Sitkiewicz T, Petersen C, et al. Minimally invasive repair of pectus excavatum--the Nuss procedure. A European multicentre experience. Eur J Pediatr Surg 2002;12:235-8.

5. Kelly RE, Goretsky MJ, Obermeyer R, et al. Twentyone years of experience with minimally invasive repair of pectus excavatum by the Nuss procedure in 1215 patients. Ann Surg 2010;252:1072-81.

6. Park HJ, Lee SY, Lee CS. Complications associated with the Nuss procedure: analysis of risk factors and suggested measures for prevention of complications. J Pediatr Surg 2004;39:391-5; discussion 391-5.

7. Pilegaard HK. Nuss technique in pectus excavatum: a mono-institutional experience. J Thorac Dis 2015;7:S172-6.

8. Sacco-Casamassima MG, Goldstein SD, Gause CD, et al. Minimally invasive repair of pectus excavatum: analyzing contemporary practice in 50 ACS NSQIP-pediatric institutions. Pediatr Surg Int 2015;31:493-9.

9. Berberich T, Haecker FM, Kehrer B, et al. Postpericardiotomy syndrome after minimally invasive 
repair of pectus excavatum. J Pediatr Surg 2004;39:e1-3.

10. Kelly RE Jr, Mellins RB, Shamberger RC, et al. Multicenter study of pectus excavatum, final report: complications, static/exercise pulmonary function, and anatomic outcomes. J Am Coll Surg 2013;217:1080-9.

11. Barsness K, Bruny J, Janik JS, et al. Delayed near-fatal hemorrhage after Nuss bar displacement. J Pediatr Surg 2005;40:e5-6.

12. Becmeur F, Ferreira CG, Haecker FM, et al. Pectus excavatum repair according to Nuss: is it safe to place a retrosternal bar by a transpleural approach, under thoracoscopic vision? J Laparoendosc Adv Surg Tech A 2011;21:757-61.

13. Gips H, Zaitsev K, Hiss J. Cardiac perforation by a pectus bar after surgical correction of pectus excavatum: case report and review of the literature. Pediatr Surg Int 2008;24:617-20.

14. Haecker FM, Berberich T, Mayr J, et al. Near-fatal bleeding after transmyocardial ventricle lesion during removal of the pectus bar after the Nuss procedure. J Thorac Cardiovasc Surg 2009;138:1240-1.

15. Lange F. Thoraxdeformitäten. In: Pfaundler $M$, Schlossmann A, editors. Handbuch der Kinderheilkunde, vol. V. Chirurgie und Orthopädie im Kindesalter. Leipzig: FCW Vogel;1910:157.

16. Haecker FM, Martinez-Ferro M. Non-surgical treatment for pectus excavatum and carinatum. In: Kolvekar SK, Pilegaard HK, editors. Chest Wall Deformities and Corrective Procedures. Switzerland: Springer International Publishing, 2016:137-60.

Cite this article as: Haecker FM, Sesia S. Vacuum bell therapy. Ann Cardiothorac Surg 2016;5(5):440-449. doi: 10.21037/ acs.2016.06.06
17. Schier F, Bahr M, Klobe E. The vacuum chest wall lifter: an innovative, nonsurgical addition to the management of pectus excavatum. J Pediatr Surg 2005;40:496-500.

18. Haecker FM, Mayr J. The vacuum bell for treatment of pectus excavatum: an alternative to surgical correction? Eur J Cardiothorac Surg 2006;29:557-61.

19. Häcker FM: Die Saugglocke nach E. Klobe zur konservativen Therapie der Trichterbrust: die Glocke als Alternative zum Bügel? Orthop. Praxis 2009;45:183-9.

20. Haecker FM. The Vacuum Bell for Treatment of Pectus Excavatum:an Effective Tool for Conservative Therapy. J Clin Anal Med 2011;2:1-4.

21. Haecker FM. The vacuum bell for conservative treatment of pectus excavatum: the Basle experience. Pediatr Surg Int 2011;27:623-7.

22. Haecker FM, Zuppinger J, Sesia SB. Die konservative Therapie der Trichterbrust mittels Vakuumtherapie. Schweiz Med Forum 2014;14:842-9.

23. Lopez M, Patoir A, Costes F, et al. Preliminary study of efficacy of cup suction in the correction of typical pectus excavatum. J Pediatr Surg 2016;51:183-7.

24. Haecker FM, Sesia S. Non-surgical treatment of pectus excavatum. J Vis Surg 2016;2:63.

25. Haecker FM, Sesia SB. Intraoperative use of the vacuum bell for elevating the sternum during the Nuss procedure. J Laparoendosc Adv Surg Tech A 2012;22:934-6.

26. Hradetzky D, Weiss S, Häcker FM, et al. A novel diagnostic tool for therapeutic monitoring during the treatment of pectus excavatum with the vacuum bell. Biomed Tech 2014;59:69-73. 